OPEN

SUBJECT AREAS:

QUANTUM

INFORMATION

QUANTUM MECHANICS

Received

5 November 2013

Accepted

23 January 2014

Published

7 February 2014

Correspondence and requests for materials should be addressed to S.J.W. (sjwu@nju.edu. cn)

\section{Reveal quantum correlation in complementary bases}

\author{
Shengiun $\mathrm{Wu}^{1,2}$, Zhihao $\mathrm{Ma}^{3,4}$, Zhihua Chen ${ }^{5,6}$ \& Sixia $\mathrm{Yu}^{2,6}$
}

${ }^{1}$ Kuang Yaming Honors School, Nanjing Univeresity, Nanjing, Jiangsu 210093, China, ${ }^{2}$ Department of Modern Physics and the Collaborative Innovation Center for Quantum Information and Quantum Frontiers, University of Science and Technology of China, Hefei, Anhui 230026, China, ${ }^{3}$ Department of Mathematics, Shanghai Jiaotong University, Shanghai 200240, China, ${ }^{4}$ Department of Physics and Astronomy, University College London, WC1E 6BT London, United Kingdom, ${ }^{5}$ Department of Science, Zhijiang College, Zhejiang University of Technology, Hangzhou 310024 , China, ${ }^{6}$ Centre for Quantum Technologies, National University of Singapore, 3 Science Drive 2, Singapore 117543, Singapore.

An essential feature of genuine quantum correlation is the simultaneous existence of correlation in complementary bases. We reveal this feature of quantum correlation by defining measures based on invariance under a basis change. For a bipartite quantum state, the classical correlation is the maximal correlation present in a certain optimum basis, while the quantum correlation is characterized as a series of residual correlations in the mutually unbiased bases. Compared with other approaches to quantify quantum correlation, our approach gives information-theoretical measures that directly reflect the essential feature of quantum correlation.

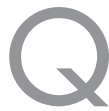

uantum physics differs significantly from classical physics in many aspects. A complete classical description of an object contains information concerning only compatible properties, while a complete quantum description of an object also contains complementary information concerning incompatible properties (see Fig. 1). This difference is also present in correlations. A classical correlation in a bipartite system involves the correlation of only a certain property, while a quantum correlation in a bipartite system also involves complementary correlations of incompatible properties. The simultaneous existence of complementary correlations together with the freedom to select which one to extract is the most important feature of quantum correlation (see Fig. 2). Schrödinger introduced the word "entanglement" to describe this peculiar feature, which was termed "spooky action at a distance" by Einstein ${ }^{1-4}$.

More recently, entangled states were defined as states that cannot be written as convex sums of product states. This precise definition is very helpful in terms of both mathematical and physical convenience, and it motivates the useful definition of the entanglement of formation. However, we now know that entanglement of formation is just one particular aspect of quantum correlation. Many measures of quantum correlation have been proposed from different perspectives, and they can be divided into two categories: entanglement measures ${ }^{5-9}$, and measures of nonclassical correlation beyond entanglement ${ }^{10-24}$.

The essential feature of quantum correlation, i.e., the simultaneous existence of complementary correlations in different bases, is also revealed by the Bell's inequalities ${ }^{25,26}$. Bell's inequalities quantify quantum correlation via expectation values of local complementary observables. Instead, we shall seek a way to directly reveal the essential feature of quantum correlation from an information-theoretical perspective. Indeed, there are several previous entropic measures of quantum correlation (such as quantum discord $D$, measurement-induced disturbance, symmetric discord, etc), which are proposed from an information-theoretical perspective. But these measures are based on the difference between quantum mutual information ${ }^{27}$ (which is assumed as the total correlation) and a certain measure of classical correlation. Here, we take a different approach and reveal the essential feature of quantum correlation directly. The genuine quantum correlation does not vanish under a change of basis, and can be characterized as the residual correlations remaining in the complementary bases.

\title{
Results
}

The idea. We begin with a comparison between the correlations in two different states:

$$
\rho_{c}=\frac{1}{2}(|00\rangle\langle 00|+| 11\rangle\langle 11|),
$$




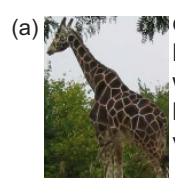

color: brown/white

height: $5 \mathrm{~m}$

weight: $300 \mathrm{~kg}$

location: Hefei

velocity: $20 \mathrm{~km} / \mathrm{h}$

(b)

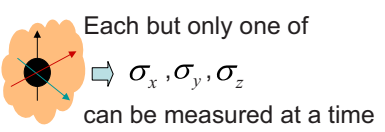

Figure 1 $\mid$ (a) A complete classical description of a classical object (a giraffe) is a simple collection of information about compatible properties, such as color, height, weight, position and velocity (the photo was taken by S.W. in Hefei animal zoo). (b) A complete quantum description (a quantum state $|\psi\rangle\rangle$ ) of a quantum system (e.g. a spin-1/2 particle) contains information about incompatible properties $\left(\sigma_{x}, \sigma_{y}, \sigma_{z}\right)$ in an intrinsic way: information about incompatible properties exists simultaneously even though only a single property can be measured at a time; and we can freely select which property to measure.

$$
|E P R\rangle=\frac{1}{\sqrt{2}}(|01\rangle-|10\rangle) .
$$

The first state has only classical correlation, which can be revealed when Alice and Bob each measure the observable $\sigma_{z}$, i.e., project their qubits onto the basis $\{|0\rangle,|1\rangle\}$. If they measure a complementary observable, $\sigma_{x}$ or $\sigma_{y}$, no correlation between their measurement results exists. The second state is the Einstein-Podolsky-Rosen (EPR) state (or the singlet state), which has both classical and quantum correlations. The classical correlation in the EPR state can be revealed when Alice and Bob each measure the same observable, e.g. $\sigma_{z}$. Moreover, this kind of correlation also exists simultaneously in complementary bases (actually, in all bases). The simultaneous existence of correlation in complementary bases is an essential feature of the genuine quantum correlation. This feature is illustrated in Fig. 2 and treated in a rigorous manner in the rest of this article.

Classical and genuine quantum correlations. For any bipartite quantum state $\rho_{A B}$, there are many measures of classical correlation $^{28}$. Here, we use the one proposed by Henderson and Vedral ${ }^{29}$, which is also used in the definition of quantum discord ${ }^{10}$. Alice selects a basis $\left\{\left|a_{i}\right\rangle_{A} \mid i=1, \cdots, d_{A}\right\}$ of her system in a $d_{A}$-dimensional Hilbert space and performs a measurement projecting her system onto the basis states. With probability $p_{i}=\operatorname{tr}_{A B}\left(\left(\left|a_{i}\right\rangle_{A}\right.\right.$ $\left.\left.\left\langle a_{i}\right| \otimes I_{B}\right) \rho_{A B}\right)$, Alice will obtain the $i$-th basis state $\left|a_{i}\right\rangle$, and Bob's system will be left in the corre sponding state $\rho_{i}^{B}={ }_{A}\left\langle a_{i}\left|\rho_{A B}\right|\right.$ $\left.a_{i}\right\rangle_{A} / p_{i}$. The Holevo quantity of the ensemble $\left\{p_{i} ; \rho_{i}^{B}\right\}$ that is prepared for Bob by Alice via her local measurement is given by $\chi\left\{\rho_{A B} \mid\left\{\left|a_{i}\right\rangle_{A}\right\}\right\}=\chi\left\{p_{i} ; \rho_{i}^{B}\right\} \equiv S\left(\sum_{i} p_{i} \rho_{i}^{B}\right)-\sum_{i} p_{i} S\left(\rho_{i}^{B}\right)$, which denotes the upper bound of Bob's accessible information about Alice's measurement result when Alice projects her system onto the basis $\left\{\left|a_{i}\right\rangle_{A}\right\}$. The classical correlation in the state $\rho_{A B}$ is defined as the maximal Holevo quantity over all local projective measurements on Alice's system:

$$
C_{1}\left(\rho_{A B}\right) \equiv \max _{\left\{\left|a_{i}\right\rangle_{A}\right\}} \chi\left\{\rho_{A B} \mid\left\{\left|a_{i}\right\rangle_{A}\right\}\right\}
$$

A basis $\left\{\left|a_{i}\right\rangle_{A}\right\}$ that achieves the maximum $C_{1}\left(\rho_{A B}\right)$ is called a $C_{1}$-basis of $\rho_{A B}$, and is denoted as $\left\{\left|\mathcal{A}_{i}^{1}\right\rangle_{A} \mid i=1, \cdots, d_{A}\right\}$. There could exist many $C_{1}$-bases for a state $\rho_{A B}$.

We consider another basis $\left\{\left|a_{j}^{2}\right\rangle_{A} \mid j=1, \cdots, d_{A}\right\}$, which is mutually unbiased to the $C_{1}$-basis $\left\{\left|\mathcal{A}_{i}^{1}\right\rangle_{A} \mid i=1, \cdots, d_{A}\right\}$ in the sense that $\left|\left\langle\mathcal{A}_{i}^{1} \mid a_{j}^{2}\right\rangle\right|=\frac{1}{\sqrt{d_{A}}}$, i.e., if the system is in a state of one basis, a projective measurement onto the mutually unbiased basis (MUB)

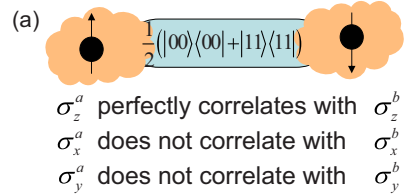

(b)

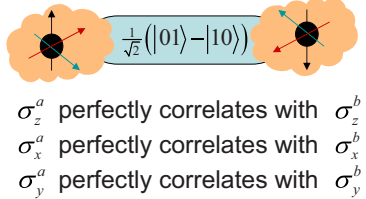

Figure $2 \mid$ (a) Classical correlation in a bipartite state reaches the maximum in a certain basis and vanishes in any complementary basis. (b) However, quantum correlation in a bipartite state contains correlations in complementary bases simultaneously; and one can freely select with which basis to read out the correlation.

will yield each basis state with the same probability. The most essential feature of quantum correlation is that when Alice performs a measurement in another basis $\left\{\left|a_{j}^{2}\right\rangle_{A} \mid j=1, \cdots, d_{A}\right\}$ that is mutually unbiased to the $C_{1}$ basis, Bob's accessible information about Alice's results, characterized by the Holevo quantity, does not vanish. This residual correlation represents genuine quantum correlation and can be used as a measure of the quantum correlation. Formally, a measure of quantum correlation $Q_{2}\left(\rho_{A B}\right)$ in the state $\rho_{A B}$ is defined as the Holevo quantity of Bob's accessible information about Alice's results, maximized over Alice's projective measurements in the bases that are mutually unbiased to a $C_{1}$-basis $\left\{\left|\mathcal{A}_{i}^{1}\right\rangle_{A} \mid i=1, \cdots, d_{A}\right\}$, and further maximized over all possible $\left\{\left|\mathcal{A}_{i}^{1}\right\rangle_{A}\right\}$ (if not unique), i.e.,

$$
Q_{2}\left(\rho_{A B}\right) \equiv \max _{\left\{\left|\mathcal{A}_{i}^{1}\right\rangle_{A}\right\}} \max _{\left\{\left|a_{j}^{2}\right\rangle_{A}\right\}} \chi\left\{\rho_{A B} \mid\left\{\left|a_{j}^{2}\right\rangle_{A}\right\}\right\} .
$$

where $\left\{\left|a_{j}^{2}\right\rangle_{A} \mid j=1, \cdots, d_{A}\right\}$ is any basis mutually unbiased to the basis $\left\{\left|\mathcal{A}_{i}^{1}\right\rangle_{A} \mid i=1, \cdots, d_{A}\right\}$. A basis $\left\{\left|a_{j}^{2}\right\rangle_{A}\right\}$ that achieves the maximum quantum correlation $Q_{2}$ in (4) is called a $Q_{2}$-basis, and is denoted as $\left\{\left|\mathcal{A}_{j}^{2}\right\rangle_{A} \mid j=1, \cdots, d_{A}\right\}$. If there is only one $C_{1}$-basis, the second maximization over the $C_{1}$-bases $\left\{\left|\mathcal{A}_{i}^{1}\right\rangle_{A}\right\}$ in (4) is not necessary. If there is more than one $C_{1}$-basis, and not all of them achieve the maximum in (4), then we redefine the $C_{1}$-bases as those that also achieve the maximum in (4). In other words, the bases (if any) that achieve the maximum in (3) but do not achieve the maximum in (4) will not be considered as $\left\{\left|\mathcal{A}_{i}^{1}\right\rangle_{A}\right\}$ any more. After this redefinition, if $\left\{\left|\mathcal{A}_{i}^{1}\right\rangle_{A}\right\}$ is still not unique, then $\left\{\left|\mathcal{A}_{j}^{2}\right\rangle_{A}\right\}$ depends on the choice of $\left\{\left|\mathcal{A}_{i}^{1}\right\rangle_{A}\right\}$. It is also obvious that $\left\{\left|\mathcal{A}_{j}^{2}\right\rangle_{A}\right\}$ is mutually unbiased to $\left\{\left|\mathcal{A}_{i}^{1}\right\rangle_{A}\right\}$.

Similar to the case of characterizing entanglement, a single quantity is not sufficient to describe the full property of quantum correlation because there could be many types of quantum correlation. Following the same line of reasoning, we can define the residual correlation in a third MUB as

$$
Q_{3}\left(\rho_{A B}\right) \equiv \max _{\left\{\left|\mathcal{A}_{i}^{1}\right\rangle_{A}\right\}} \max _{\left\{\left|\mathcal{A}_{j}^{2}\right\rangle_{A}\right\}} \max _{\left\{\left|a_{k}^{3}\right\rangle_{A}\right\}} \chi\left\{\rho_{A B} \mid\left\{\left|a_{k}^{3}\right\rangle_{A}\right\}\right\},
$$

where $\left\{\left|a_{k}^{3}\right\rangle_{A} \mid k=1, \cdots, d_{A}\right\}$ is any basis mutually unbiased to both $\left\{\left|\mathcal{A}_{i}^{1}\right\rangle_{A}\right\}$ and $\left\{\left|\mathcal{A}_{j}^{2}\right\rangle_{A}\right\}$. An optimum basis $\left\{\left|a_{k}^{3}\right\rangle_{A}\right\}$ to achieve the maximum in (5) is called a $Q_{3}$-basis, and is denoted as $\left\{\left|\mathcal{A}_{k}^{3}\right\rangle_{A} \mid k=\right.$ $\left.1, \cdots, d_{A} \cdot\right\}$. Similarly, we redefine the $Q_{2}$-bases $\left\{\left|\mathcal{A}_{j}^{2}\right\rangle_{A}\right\}$ as those that are optimum in both (4) and (5), and further redefine the $C_{1^{-}}$ bases $\left\{\left|\mathcal{A}_{i}^{1}\right\rangle_{A}\right\}$ as those that are optimum in (3), (4) and (5). 
Suppose in this manner that we can define $M$ quantities for the measures of correlation, which are conveniently written as a single correlation vector $\vec{C} \equiv\left(C_{1}, Q_{2}, Q_{3}, \cdots, Q_{M}\right)$ for the state $\rho_{A B}$. The number $M$ cannot be greater than the number of MUBs that exist in the $d_{A}$-dimensional Hilbert space. The first quantity $C_{1}$ denotes the maximal classical correlation present in the state $\rho_{A B}$, which can be revealed when Alice performs a measurement of her system in a $C_{1}$-basis $\left\{\left|\mathcal{A}_{i}^{1}\right\rangle_{A} \mid i=1, \cdots, d_{A}\right\}$. As classical correlation will vanish when measured in a mutually unbiased basis, all of the other quantities describe genuine quantum types of correlation. The second quantity $Q_{2}$ denotes the maximal genuine quantum correlation, and $\left\{\left|\mathcal{A}_{j}^{2}\right\rangle_{A} \mid j=1, \cdots, d_{A}\right\}$ denotes an optimum basis to reveal this correlation. The third quantity $Q_{3}$ denotes another type of genuine quantum correlation, and $\left\{\left|\mathcal{A}_{k}^{3}\right\rangle_{A} \mid j=1, \cdots, d_{A}\right\}$ denotes a basis to reveal the second type of quantum correlation.

The splitting of the correlation vector as a single classical component $\left(C_{1}\right)$ and several quantum components $\left(Q_{2}, \cdots, Q_{M}\right)$ is not artificial, in fact, this splitting captures the essential difference between classical correlation and quantum correlation. The quantity $C_{1}$ represents the maximal amount of correlation available in a single basis $\left(\left\{\left|\mathcal{A}_{i}^{1}\right\rangle_{A}\right\}\right)$. The quantity $Q_{2}$ represents the maximal amount of correlation that is available not only in the first basis $\left\{\left|\mathcal{A}_{i}^{1}\right\rangle_{A}\right\}$ but also in a second complementary basis $\left\{\left|\mathcal{A}_{j}^{2}\right\rangle_{A}\right\}$ (we know $C_{1} \geq Q_{2} \geq Q_{3} \geq \cdots$ from the definition of these quantities). And $Q_{3}$ represents the maximal amount of correlation available not only in $\left\{\left|\mathcal{A}_{i}^{1}\right\rangle_{A}\right\}$ and $\left\{\left|\mathcal{A}_{j}^{2}\right\rangle_{A}\right\}$, but also in a third MUB $\left\{\left|\mathcal{A}_{k}^{3}\right\rangle_{A}\right\}$. The amount $Q_{3}$ of correlation exists in 3 MUBs while the amount $Q_{2}$ of correlation may only exist in 2 MUBs. Thus, $Q_{3}$ represents the amount of correlation with a higher level of quantumness than that of $Q_{2}$, and may have more practical advantages when 3 MUBs are necessarily used (e.g. entanglement-based QKD via 6-state protocol).

It should be pointed out that the maximum number of MUBs that exist in a $d_{A}$-dimensional Hilbert space is not known for the general case. When $d_{A}$ is a power of a prime number, a full set of $d_{A}+1$ MUBs exists; for other cases, there may not exist $d_{A}+1$ MUBs. For example, when $d_{A}=6$, only 3 MUBs have been found yet, while 3 is much less than $d_{A}+1=7$. Many interesting works can be found on the existence of MUBs in the literature ${ }^{30-34}$. Since there exist at least 3 MUBs for any integer $d_{A} \geq 2$, the quantities $C_{1}, Q_{2}$, and $Q_{3}$ are welldefined for any $d_{A} \geq 2$. In many cases, we are interested in combined systems of qubits with $d_{A}$ being a power of 2, thus, $d_{A}+1$ MUBs exist and quantities $C_{1}, Q_{2}, \cdots, Q_{d_{A}+1}$ are all well-defined. For an arbitrary $d_{A}$-dimensional Hilbert space, we don't make assumptions about the maximal number of MUBs that exist, we only assume that $M$ MUBs are available, where $M$ is less or equal to the maximal number of MUBs that exist. In many cases, we only discuss the first 3 elements $\left(C_{1}, Q_{2}\right.$, and $\left.Q_{3}\right)$ of the correlation vector for simplicity.

Examples. Now, we shall calculate the correlation vector for several families of bipartite states, and see how these measures in terms of MUBs are well justified as measures of classical and genuine quantum correlations.

For a bipartite pure state written in the Schmidt basis, $|\psi\rangle_{A B}=\sum_{i} \sqrt{\lambda_{i}}\left|a_{i}\right\rangle\left|b_{i}\right\rangle$, the maximal classical correlation can be revealed when Alice performs her measurement onto her Schmidt basis $\left\{\left|a_{i}\right\rangle\right\}$; thus, one immediately has $C_{1}=S\left(\rho_{B}\right)=\sum_{i}-\lambda_{i} \log _{2} \lambda_{i}$. If Alice chooses another basis $\left\{\left|a_{i}^{\prime}\right\rangle\right\}$, whenever she obtains a particular measurement result, Bob will be left with a pure state. Therefore, one can easily obtain the maximal true quantum correlation $Q_{2}=S\left(\rho_{B}\right)=C_{1}$; any other basis will yield the same amount of quantum correlation. Therefore, the correlation vector for a bipartite pure state $|\psi\rangle_{A B}$ is given as $\vec{C}=\left(S\left(\rho_{B}\right), S\left(\rho_{B}\right), \cdots, S\left(\rho_{B}\right)\right)$. The correlation vector exhibits a unique feature of the correlations in a pure state: the classical correlation is equal to the quantum correlation revealed in any basis, and both values are equal to the von Neumann entropy of the reduced density matrix on either side, which is the usual measure of entanglement in a pure state.

A classical-quantum (CQ) state is a bipartite state that can be written as

$$
\rho^{c q}=\sum_{i} q_{i}|i\rangle\langle i| \otimes \sigma_{i},
$$

where $\left\{q_{i}\right\}$ is a probability distribution, $\left\{|i\rangle \mid i=0,1, \cdots, d_{A}-1\right\}$ is a basis of system $A$ in a $d_{A}$-dimensional Hilbert space, and $\left\{\sigma_{i}\right\}$ is a set of density matrices of system $\mathrm{B}$. The maximal classical correlation is revealed when Alice performs her measurement in the basis $\{|i\rangle\}^{12}$; thus, the maximal classical correlation in the CQ state $\rho^{c q}$ is given by $C_{1}=\chi\left\{q_{i} ; \sigma_{i}\right\}=S\left(\sum_{i} q_{i} \sigma_{i}\right)-\sum_{i} q_{i} S\left(\sigma_{i}\right)$. To calculate the amount of quantum correlation, Alice projects her system onto another basis $\left\{\left|a_{j}^{2}\right\rangle\right\}$ that is mutually unbiased to the optimum basis $\{|i\rangle\}$ for classical correlation. From $\left|\left\langle i \mid a_{j}^{2}\right\rangle\right|^{2}=\frac{1}{d_{A}}$, we have

$$
\begin{aligned}
\left\langle a_{j}^{2}\left|\rho^{c q}\right| a_{j}^{2}\right\rangle & =\sum_{i} q_{i}\left\langle a_{j}^{2}|(|i\rangle\langle i|)| a_{j}^{2}\right\rangle \sigma_{i} \\
& =\frac{1}{d_{A}} \sum_{i} q_{i} \sigma_{i}=\frac{1}{d_{A}} \rho_{B} .
\end{aligned}
$$

For each different result $j$ that Alice obtains, Bob is left with the same state $\rho_{B}=\sum_{i} q_{i} \sigma_{i}$; thus, Bob's state has no correlation with Alice's result, and we immediately have $Q_{2}=Q_{3} \cdots=0$ according to the definitions of these quantities. Hence, for a CQ state, the correlation vector is given as $\vec{C}=\left(C_{1}, 0, \cdots, 0\right)$. The only correlation present in a CQ state is the classical correlation, and the quantum correlation in any MUB vanishes!

Next, we consider the Werner states of a $d \times d$ dimensional system $^{35}$

$$
\rho_{w}=\frac{1}{d(d-\alpha)}(I-\alpha P),
$$

where $-1 \leq \alpha \leq 1, I$ is the identity operator in the $d^{2}$-dimensional Hilbert space, and $P=\sum_{i, j=1}^{d}|i\rangle\langle j|\otimes| j\rangle\langle i|$ is the operator that exchanges $\mathrm{A}$ and $\mathrm{B}$. Because the Werner states are invariant under a unitary transformation of the form $U \otimes U$, the maximal classical correlation can be revealed when Alice simply projects her system onto the basis states $\{|i\rangle\}$. With probability $p_{i}=\frac{1}{d}$, Alice will obtain the $i$-th basis state $|i\rangle$, and Bob will be left with the state $\rho_{i}^{B}=A\left\langle i\left|\rho_{A B}\right| i\right\rangle_{A} / p_{i}=\frac{1}{d-\alpha}(I-\alpha|i\rangle\langle i|)$. It is straightforward to show that $C_{1}=\chi\left\{p_{i} ; \rho_{i}^{B}\right\}=\log _{2}\left(\frac{d}{d-\alpha}\right)+\frac{1-\alpha}{d-\alpha} \log _{2}(1-\alpha) \equiv \chi_{w}$. Due to the symmetry of the Werner states, it is not difficult to demonstrate that $Q_{2}=Q_{3}=\cdots=C_{1}=\chi_{w}$. Therefore, for the Werner state $\rho_{w}$, the correlation vector is given by $\vec{C}=\left(\chi_{w}, \chi_{w}, \cdots, \chi_{w}\right)$. The maximal quantum correlation in a Werner state can be revealed in any basis, and it is equal to the maximal classical correlation $C_{1}$. However, the correlation vector of a Werner state is different from that of a pure state because $C_{1} \leq S\left(\rho_{B}\right)=\log _{2} d$. The inequality becomes an equality only when $d=2$ and $\alpha=1$, in which case, the Werner state becomes a pure state $\rho_{w}=|E P R\rangle\langle E P R|$. For the Werner states, the symmetric discord is equal to the quantum discord $D^{12}$ when Alice's measurement is restricted to projective mea- 

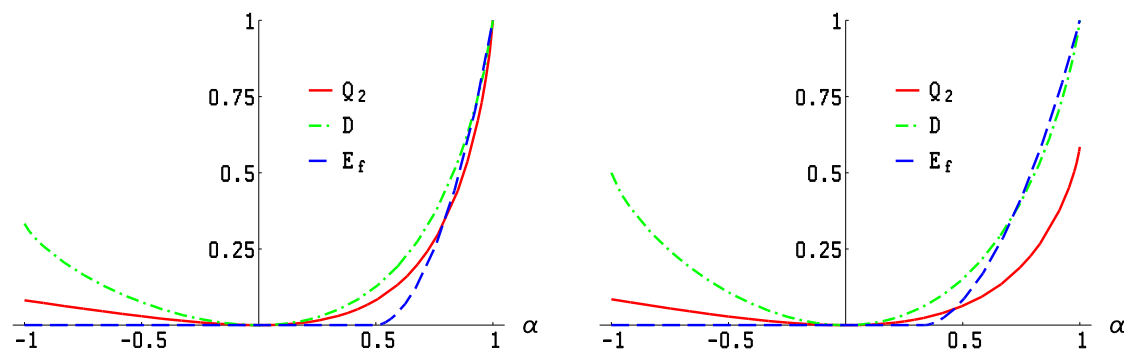

Figure $3 \mid$ Three measures of quantum correlation for the Werner states as functions of $\alpha$ when $d=2$ (left) and $d=3$ (right). The red curve represents our measure $Q_{2}$, the green curve represents the quantum discord $D$ and the blue curve represents the entanglement of formation $E_{f}$.

surements. The entanglement of formation $E_{f}$ for the Werner states is given as $E_{f}\left(\rho_{w}\right)=h\left(\frac{1}{2}\left(1+\sqrt{1-\left[\max \left(0, \frac{d \alpha-1}{d-\alpha}\right)\right]^{2}}\right)\right)$, with $h(x) \equiv-x \log _{2} x-(1-x) \log _{2}(1-x)^{36}$. The three different measures of quantum correlation, i.e., our measure of maximal quantum correlation $Q_{2}$, the quantum discord $D$ and the entanglement of formation, are illustrated in Fig. 3 for comparison. From this figure, we see that the curve for entanglement of formation intersects the other two curves; thus, $E_{f}$ can be larger or smaller than $Q_{2}(D)$.

As the last example, we consider a family of two-qubit states, where the reduced density matrices of both qubits are proportional to the identity operator. Such a state can be written in terms of Pauli matrices,

$$
\rho_{A B}=\frac{1}{4}\left(I_{2} \otimes I_{2}+\sum_{j, k=1}^{3} w_{j k} \sigma_{j} \otimes \sigma_{k}\right)
$$

where $I_{2}$ is the identity operator in the two-dimensional Hilbert space of a qubit, and $w_{j k}$ are real numbers that satisfy certain conditions to ensure the positivity of the matrix in (9). These two-qubit states can be transformed by a local unitary transformation (that does not change the correlations) to the following form:

$$
\sigma_{A B}=\frac{1}{4}\left(I_{2} \otimes I_{2}+\sum_{j=1}^{3} r_{j} \sigma_{j} \otimes \sigma_{j}\right)
$$

which is equivalent to the Bell-diagonal states. To ensure the positivity of the matrix in (10), the real vector $\vec{r}=\left(r_{1}, r_{2}, r_{3}\right)$ must lie inside or on the boundary of the regular tetrahedron that is the convex hull of the four points: $(-1,-1,-1),(-1,1,1),(1,-1,1)$ and $(1,1,-1)$ (which are the four Bell states). The singular values of the matrix $w_{j k}$ are given by $\left|r_{j}\right|$. We rearrange the three numbers $\left\{r_{1}, r_{2}, r_{3}\right\}$ according to their absolute values and denote the rearranged set as $\left\{\bar{r}_{1}, \bar{r}_{2}, \bar{r}_{3}\right\}$ such that $\left|\bar{r}_{1}\right| \geq\left|\bar{r}_{2}\right| \geq\left|\bar{r}_{3}\right|$.

In the Methods, we prove that the correlation vector of the state in (9) is given by $\vec{C}=\left(\chi_{1}, \chi_{2}, \chi_{3}\right)$, where $\chi_{j}=1-h\left(\frac{1+\left|\bar{r}_{j}\right|}{2}\right)$ with $h(x) \equiv$

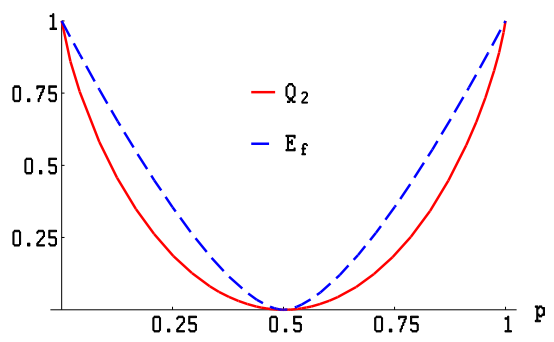

$-x \log _{2} x-(1-x) \log _{2}(1-x)$. To have some intuition of this result, we consider some special classes of states with only one parameter. When $r_{1}=r_{2}=r_{3}=-\frac{\alpha}{2-\alpha}$ with $-1 \leq \alpha \leq 1$, the states in (10) become the Werner states for $d=2$ in (6). When $r_{1}=r_{2}=1-$ $2 p$ and $r_{3}=-1$ with $0 \leq p \leq 1$, the states in (10) become $\rho_{1}=p\left|\psi^{-}\right\rangle$ $\left\langle\psi^{-}|+(1-p)| \psi^{+}\right\rangle\left\langle\psi^{+}\right|$; we obtain $C_{1}=1$ and $Q_{2}=Q_{3}=1-h(p)$. When $r_{1}=1-2 p$ and $r_{2}=r_{3}=-p$, the states in (10) become $\rho_{2}=p\left|\psi^{-}\right\rangle\left\langle\psi^{-}\right|+\frac{1-p}{2}\left(\left|\psi^{+}\right\rangle\left\langle\psi^{+}|+| \phi^{+}\right\rangle\left\langle\phi^{+}\right|\right)$; we have $C_{1}=$ $\max \left\{1-h(p), 1-h\left(\frac{1+p}{2}\right)\right\}, Q_{2}=1-h\left(\frac{1+p}{2}\right)$ and $Q_{3}=\min$ $\left\{1-h(p), 1-h\left(\frac{1+p}{2}\right)\right\}$. Here, $\left|\psi^{-}\right\rangle=|E P R\rangle=\frac{1}{\sqrt{2}}(|01\rangle-|10\rangle)$, $\left|\psi^{+}\right\rangle=\frac{1}{\sqrt{2}}(|01\rangle+|10\rangle)$ and $\left|\phi^{+}\right\rangle=\frac{1}{\sqrt{2}}(|00\rangle+|11\rangle)$. Our measure of quantum correlation $Q_{2}$ is compared with the quantum discord $D$ and the entanglement of formation $E_{f}$ for $\rho_{1}$ and $\rho_{2}$ in Fig. 4.

Inequality relations between correlation measures. It is not difficult to show that the relation $Q_{2} \leq D$ holds for the Werner states, and for all the example states considered in this article. However, it is not clear whether this inequality holds for any bipartite states. If $Q_{2} \leq D$ holds for any bipartite states, then one can easily have $C_{1}+Q_{2} \leq S(A: B)$ where $S(A: B)=S\left(\rho_{A}\right)+S\left(\rho_{B}\right)-$ $S\left(\rho_{A B}\right)$ denotes the quantum mutual information.

Nevertheless, we can prove the following inequality:

$$
C_{1}+Q_{2} \leq H_{1}+H_{2}+S\left(\rho_{B}\right)-S\left(\rho_{A B}\right)-\log _{2} d_{A}
$$

where $H_{\gamma}(\gamma=1,2)$ denotes the Shannon entropy of the probability distribution $\left\{p_{i}^{(\gamma)}\right\}$ obtained by the measurement on system $\mathrm{A}$ in the basis $\left\{\left|\mathcal{A}_{i}^{\gamma}\right\rangle_{A} \mid i=1, \cdots, d_{A}\right\}$. The proof is given in the Methods. Since $H_{\gamma} \leq \log _{2} d_{A}$, one immediately has

$$
C_{1}+Q_{2} \leq S\left(\rho_{B}\right)-S\left(\rho_{A B}\right)+\log _{2} d_{A} .
$$

As $C_{1}$ and $Q_{2}$ are the two largest elements in the correlation vector, when they are replaced by correlations in any two MUBs, inequalities (11) and (12) still hold.

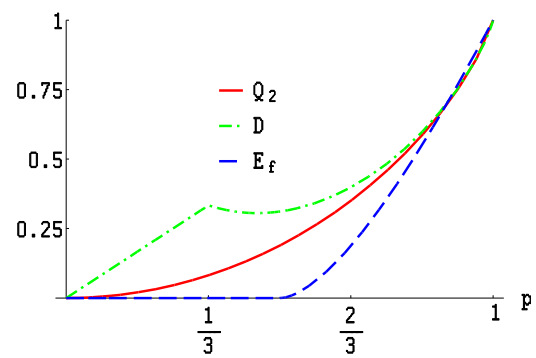

Figure $4 \mid$ Different measures of quantum correlation for two special classes of states: $\rho_{1}=p\left|\psi^{-}\right\rangle\left\langle\psi^{-}|+(1-p)| \psi^{+}\right\rangle\left\langle\psi^{+}\right|$(left) and $\rho_{2}=p\left|\psi^{-}\right\rangle\left\langle\psi^{-}\right|+\frac{1-p}{2}\left(\left|\psi^{+}\right\rangle\left\langle\psi^{+}|+| \phi^{+}\right\rangle\left\langle\phi^{+}\right|\right)$(right). In each figure, the red curve represents our measure $Q_{2}$, the green curve represents the quantum discord $D$, and the blue curve represents the entanglement of formation $E_{f}$. In the left figure, the green curve is not shown because $D=Q_{2}$ for $\rho_{1}$. 


\section{Discussion}

Our measures of quantum correlation provide a natural way to quantify the "spooky action at a distance", and directly reveal the essential feature of the genuine quantum correlation, i.e., the simultaneous existence of correlations in complementary bases. This feature enables quantum key distribution with entangled states, since the quantum correlation that exists simultaneously in two $(k)$ MUBs, which is quantified by $Q_{2}\left(Q_{k}\right)$, is the resource for entanglementbased QKD via two $(k)$ MUBs. Quantitative relation between the genuine quantum correlation and the secret key fraction in QKD could be studied in further work.

All the measures considered above are not symmetric with respect to the exchange of systems A and B, as only system A's bases are considered to reveal the correlation. Symmetric measures and a symmetric correlation vector are also introduced and discussed in the Methods. A further study of the relation between the symmetric correlation vector and the symmetric discord ${ }^{12}$ could reveal the difference between these measures in practical applications.

There are some open questions. Are our measures $\left(Q_{2}, Q_{3}\right)$ of genuine quantum correlation additive? How do they behave under some natural operations (for example, Alice adds an ancilla)? Do our measures $\left(Q_{2}, Q_{3}\right)$ behave like the entanglement measures that do not increase under local operations and classical communication $(\mathrm{LOCC})^{9}$, or more like the measures of nonclassical correlation beyond entanglement (for example, quantum discord) that could increase under LOCC $^{38}$ ? We hope that further investigations will unveil these mysteries.

\section{Methods}

Proof of the inequality (11). Here we prove inequality (11) in the main text. Let $\left\{p_{i}^{(\gamma)}\right\}(\gamma=1,2)$ denote the probability distribution obtained by the measurement on system $\mathrm{A}$ in the basis $\left\{\left|\mathcal{A}_{i}^{\gamma}\right\rangle_{A} \mid i=1, \cdots, d_{A}\right\}$, i.e., $p_{i}^{(\gamma)}=\operatorname{tr}_{A B}$ $\left(\left(\left|\mathcal{A}_{i}^{\gamma}\right\rangle\left\langle\mathcal{A}_{i}^{\gamma}\right| \otimes I\right) \rho_{A B}\right)$. Let $H_{\gamma}(\gamma=1,2)$ denote the Shannon entropy of the probability distribution, $H_{\gamma}=\sum_{i=1}^{d_{A}}-p_{i}^{(\gamma)} \log _{2} p_{i}^{(\gamma)}$. Here, the basis $\left\{\left|\mathcal{A}_{i}^{1}\right\rangle_{A} \mid i=1, \cdots, d_{A}\right\}$ is the optimum basis for measurement on system $A$ to achieve the maximum classical correlation $C_{1}$, and the basis $\left\{\left|\mathcal{A}_{i}^{2}\right\rangle_{A}\right\}$ is the optimum basis to achieve $Q_{2}$ among the bases that are mutually unbiased to $\left\{\left|\mathcal{A}_{i}^{1}\right\rangle_{A}\right\}$. However, the proof below only requires that $\left\{\left|\mathcal{A}_{i}^{i}\right\rangle_{A}\right\}$ and $\left\{\left|\mathcal{A}_{i}^{2}\right\rangle_{A}\right\}$ are mutually unbiased to each other.

Let $\rho^{(\gamma)}=\sum_{i=1}^{d_{A}}\left|\mathcal{A}_{i}^{\gamma}\right\rangle\left\langle\mathcal{A}_{i}^{\gamma}\right| \otimes p_{i}^{(\gamma)} \rho_{i}^{(\gamma)}$ with $\gamma=1,2$, where

$\rho_{i}^{(\gamma)}=\left\langle\mathcal{A}_{i}^{\gamma}\left|\rho_{A B}\right| \mathcal{A}_{i}^{\gamma}\right\rangle / p_{i}^{(\gamma)}$.

The uncertainty relation ${ }^{37}$ gives

$$
S\left(\left\{\left|\mathcal{A}_{i}^{1}\right\rangle\right\} \mid B\right)+S\left(\left\{\left|\mathcal{A}_{i}^{2}\right\rangle\right\} \mid B\right) \geq \log _{2} d_{A}+S(A \mid B)
$$

where $S(A \mid B)=S\left(\rho_{A B}\right)-S\left(\rho_{B}\right)$, and $S\left(\left|\mathcal{A}_{i}^{\gamma}\right\rangle \mid B\right)=S\left(\rho^{(\gamma)}\right)-S\left(\rho_{B}\right)(\gamma=1,2)$. As $\rho^{(\gamma)}$ is a CQ state, one can show that $S\left(\rho^{(\gamma)}\right)=H_{\gamma}+\sum_{i} p_{i}^{(\gamma)} S\left(\rho_{i}^{(\gamma)}\right)$. Therefore,

$$
\begin{aligned}
& H_{1}+\sum_{i} p_{i}^{(1)} S\left(\rho_{i}^{(1)}\right)-S\left(\rho_{B}\right) \\
+ & H_{2}+\sum_{i} p_{i}^{(2)} S\left(\rho_{i}^{(2)}\right)-S\left(\rho_{B}\right) \\
\geq & \log _{2} d_{A}+S\left(\rho_{A B}\right)-S\left(\rho_{B}\right)
\end{aligned}
$$

As $C_{1}=S\left(\rho_{B}\right)-\sum_{i} p_{i}^{(1)} S\left(\rho_{i}^{(1)}\right)$ and $Q_{2}=S\left(\rho_{B}\right)-\sum_{i} p_{i}^{(2)} S\left(\rho_{i}^{(2)}\right)$, we immediately have

$$
C_{1}+Q_{2} \leq H_{1}+H_{2}+S\left(\rho_{B}\right)-S\left(\rho_{A B}\right)-\log _{2} d_{A}
$$

which completes the proof of the inequality.

Calculation of the correlation vector for the states in (9). In this paragraph, we shall demonstrate that the correlation vector of the state in (9) is given by $\vec{C}=\left(\chi_{1}, \chi_{2}, \chi_{3}\right)$, where $\chi_{j}=1-h\left(\frac{1+\left|\bar{r}_{j}\right|}{2}\right)$ with $h(x) \equiv-x \log _{2} x-(1-x) \log _{2}(1-x)$. We perform the calculation in the transformed basis, with the states rewritten in Eq. (10). Without loss of generality, we can suppose the numbers $r_{j}$ are already arranged according to $\left|r_{1}\right|$ $\geq\left|r_{2}\right| \geq\left|r_{3}\right|$; then, we only need to prove that $\vec{C}=\left(\chi_{1}, \chi_{2}, \chi_{3}\right)$, where $\chi_{j}=1-h\left(\frac{1+\left|r_{j}\right|}{2}\right)$. A projective measurement performed on qubit A can be written $P_{ \pm}^{A}=\frac{1}{2}\left(I_{2} \pm \vec{n} \cdot \vec{\sigma}\right)$, parameterized by the unit vector $\vec{n}$. We have

$$
p \pm \rho_{ \pm}^{B} \equiv \operatorname{Tr}_{A}\left(P_{ \pm}^{A} \rho_{A B}\right)=\frac{1}{2} \cdot \frac{1}{2}\left(I_{2} \pm \sum_{j} n_{j} r_{j} \sigma_{j}\right) .
$$

When Alice obtains \pm , qubit B will be in the corresponding states $\rho_{ \pm}^{B}=\frac{1}{2}\left(I_{2}+\sum_{j} n_{j} r_{j} \sigma_{j}\right)$, each occurring with probability $\frac{1}{2}$. The entropy $S\left(\rho_{ \pm}^{B}\right)$ reaches its minimum value $h\left(\frac{1+\left|r_{1}\right|}{2}\right)$ when $\vec{n}=(1,0,0)$. From $\rho_{B}=p+\rho_{+}^{B}+p-\rho_{-}^{B}=\frac{1}{2} I_{2}$ and $S\left(\rho_{B}\right)=1$, we immediately have $C_{1}=1-h\left(\frac{1+\left|r_{1}\right|}{2}\right)$. The basis for Alice's projection $P_{ \pm}^{A}=\frac{1}{2}\left(I_{2}+\vec{n}^{\prime} \cdot \vec{\sigma}\right)$ in the definition of $Q_{2}$ must be mutually unbiased to the basis parameterized by $\vec{n}=(1,0,0)$; therefore, the unit vector $\vec{n}^{\prime}$ must be in the form $\vec{n}^{\prime}=\left(0, n_{2}, n_{3}\right)$. The maximum in the definition of $Q_{2}$ is reached when $\vec{n}^{\prime}=(0,1,0)$, and thus a calculation similar to that for $C_{1}$ yields $Q_{2}=1-h\left(\frac{1+\left|r_{2}\right|}{2}\right)$. For a qubit system, three MUBs exist. We can reveal the quantum correlation in another (the last) MUB, which corresponds to the case $\vec{n}^{\prime \prime}=(0,0,1)$. We easily obtain $Q_{3}=1-h\left(\frac{1+\left|r_{3}\right|}{2}\right)$. For the general case in which the numbers $r_{j}$ do not follow $\left|r_{1}\right| \geq\left|r_{2}\right| \geq\left|r_{3}\right|$, a similar argument yields $\vec{C}=\left(\chi_{1}, \chi_{2}, \chi_{3}\right)$ with $\chi_{j}=1-h\left(\frac{1+\left|\bar{r}_{j}\right|}{2}\right)$.

Symmetric correlation vector. The correlation vector defined in the main text relies on a special choice of the measure of classical correlation; it is not symmetric with respect to exchange of A and B. Here, we consider an alternative definition of the correlation vector, which is symmetric with respect to the exchange of $\mathrm{A}$ and $\mathrm{B}$.

For any bipartite quantum state $\rho_{A B}$, Alice chooses a basis $\left\{\left|a_{i}\right\rangle_{A} \mid i=1, \cdots, d_{A}\right\}$ of her system in a $d_{A}$-dimensional Hilbert space and Bob chooses a basis $\left\{\left|b_{i}\right\rangle_{A} \mid i=1, \cdots, d_{B}\right\}$ of his system in a $d_{B}$-dimensional Hilbert space, and each one performs a measurement projecting his/her system onto the corresponding basis states. With probability $p_{i j}=\operatorname{tr}_{A B}\left(\left(\left|a_{i}\right\rangle_{A}\left\langle a_{i}|\otimes| b_{j}\right\rangle_{A}\left\langle b_{j}\right|\right) \rho_{A B}\right)$, Alice and Bob will obtain the $i$-th and $j$-th results, respectively. The correlation of their measurement results is well characterized by the classical mutual information:

$$
I\left\{p_{i j}\right\}=H\left\{p_{i}^{a}\right\}+H\left\{p_{j}^{b}\right\}-H\left\{p_{i j}\right\},
$$

where $H\left\{p_{k}\right\}$ is the Shannon entropy of the probability distribution $\left\{p_{k}\right\}$, and $p_{i}^{a}=\sum_{i} p_{i j}$ and $p_{j}^{b}=\sum_{i} p_{i j}$ are the marginal probability distributions. In other words, $H\left\{p_{i}^{a}\right\}=\sum_{i}-p_{i}^{a} \log _{2} p_{i}^{a}$, and $H\left\{p_{i j}\right\}=\sum_{i j}-p_{i j} \log _{2} p_{i j}$.

The symmetric measure of classical correlation $C_{1}^{s}$ in the state $\rho_{A B}$ is defined as the maximal classical mutual information of the local measurement results, maximized over all local bases for both sides, i.e.,

$$
C_{1}^{s}\left(\rho_{A B}\right)=\max _{\left\{\left|a_{i}\right\rangle \otimes\left|b_{j}\right\rangle\right\}} I\left\{p_{i j}\right\} .
$$

The symmetric measure of classical correlation was discussed in $^{12}$ (where the notation $I_{\text {max }}$ was used). A product basis $\left\{\left|a_{i}^{1}\right\rangle \otimes\left|b_{j}^{1}\right\rangle \mid i=1, \cdots, d_{A}, j=1, \cdots, d_{B}\right\}$ that achieves the maximum in (18) is called a $C_{1}^{s}$-basis, and is denoted as

$\left\{\left|\mathcal{A}_{i}^{1}\right\rangle \otimes\left|\mathcal{B}_{j}^{1}\right\rangle \mid i=1, \cdots, d_{A}, j=1, \cdots, d_{B}\right\}$.

The symmetric measure of maximal quantum correlation $Q_{2}^{s}$ is defined as the maximal residual correlation over all local bases that are mutually unbiased to a $C_{1-}^{s}$ basis $\left\{\left|\mathcal{A}_{i}^{1}\right\rangle \otimes\left|\mathcal{B}_{j}^{1}\right\rangle\right\}$, further maximized over all possible $C_{1}^{s}$-bases (if not unique), i.e.,

$$
Q_{2}^{s}\left(\rho_{A B}\right)=\max _{\left\{\left|\mathcal{A}_{i}^{1}\right\rangle \otimes\left|\mathcal{B}_{j}^{1}\right\rangle\right\}} \max _{\left\{\left|a_{i}^{2}\right\rangle \otimes\left|b_{j}^{2}\right\rangle\right\}} I\left\{p_{i j}^{\prime}\right\},
$$

where $\left\{\left|a_{i}^{2}\right\rangle\right\}\left(\left\{\left|b_{j}^{2}\right\rangle\right\}\right)$ is mutually unbiased to $\left\{\left|\mathcal{A}_{i}^{1}\right\rangle_{A}\right\}\left(\left\{\left|\mathcal{B}_{j}^{1}\right\rangle_{B}\right\}\right)$ and $p_{i j}^{\prime}=\operatorname{tr}_{A B}\left(\left(\left|a_{i}^{2}\right\rangle_{A}\left\langle a_{i}^{2}|\otimes| b_{j}^{2}\right\rangle_{A}\left\langle b_{j}^{2}\right|\right) \rho_{A B}\right)$. A basis $\left\{\left|a_{i}^{2}\right\rangle \otimes\left|b_{j}^{2}\right\rangle\right\}$ that achieves the maximum in (19) is called a $Q_{2}^{s}$-basis, and is denoted as $\left\{\left|\mathcal{A}_{i}^{2}\right\rangle \otimes\left|\mathcal{B}_{j}^{2}\right\rangle\right\}$. We redefine the $C_{1}^{s}$-bases $\left\{\left|\mathcal{A}_{i}^{1}\right\rangle \otimes\left|\mathcal{B}_{j}^{1}\right\rangle\right\}$ as the bases that achieve the maximum in (18) as well as the maximum in (19).

Similarly, $Q_{3}^{s}$ denotes the residual quantum correlation in a third complementary basis that is mutually unbiased to both $\left\{\left|\mathcal{A}_{i}^{1}\right\rangle \otimes\left|\mathcal{B}_{j}^{1}\right\rangle\right\}$ and $\left\{\left|\mathcal{A}_{i}^{2}\right\rangle \otimes\left|\mathcal{B}_{j}^{2}\right\rangle\right\}$. In this 
manner, we have an alternative correlation vector $\vec{C}_{s}=\left(C_{1}^{s}, Q_{2}^{s}, Q_{3}^{s}, \cdots, Q_{M}^{s}\right)$, which is symmetric with respect to the change of $\mathrm{A}$ and $\mathrm{B}$.

Because the Holevo bound is an upper bound of accessible classical mutual information, we immediately know from the above definitions that the asymmetric correlation vector $\vec{C}$ is an upper bound of the symmetric correlation vector $\vec{C}_{s}$ for each component, i.e., $C_{1} \geq C_{1}^{s}, Q_{2} \geq Q_{2}^{s}, \cdots, Q_{M} \geq Q_{M}^{s}$. It is not difficult to demonstrate that the asymmetric correlation vector $\vec{C}$ actually coincides with the symmetric correlation vector $\vec{C}_{s}$ (i.e., $\vec{C}=\vec{C}_{s}$ ) for the CQ states, the Werner states and the twoqubit states in Eq. (9).

1. Einstein, A., Podolsky, B. \& Rosen, N. Can quantum-mechanical description of physical reality be considered complete? Phys. Rev. 47, 777-780 (1935).

2. Schrödinger, E. Die gegenwärtige Situation in der Quantenmechanik. Naturwissenschaften 23, 807-812 (1935).

3. Schrödinger, E. \& Born, M. Discussion of probability relations between separated systems. Math. Proc. Camb. Phil. Soc. 31, 555-563 (1935).

4. Einstein, A., Born, M. \& Born, H. The Born-Einstein Letters: correspondence between Albert Einstein and Max and Hedwig Born from 1916 to 1955 (Walker, New York, 1971)

5. Bennett, C. H., Bernstein, H. J., Popescu, S. \& Schumacher, B. Concentrating partial entanglement by local operations. Phys. Rev. A 53, 2046-2052 (1996).

6. Bennett, C. H., DiVincenzo, D. P., Smolin, J. A. \& Wootters, W. K. Mixed-state entanglement and quantum error correction. Phys. Rev. A 54, 3824-3851 (1996).

7. Vedral, V., Plenio, M. B., Rippin, M. A. \& Knight, P. L. Quantifying Entanglement. Phys. Rev. Lett. 78, 2275-2279 (1997).

8. Christandl, M. \& Winter, A. "Squashed Entanglement"- An Additive Entanglement Measure. J. Math. Phys. 45, 829-840 (2004).

9. Horodecki, R., Horodecki, P., Horodecki, M. \& Horodecki, K. Quantum entanglement. Rev. Mod. Phys. 81, 65-942 (2009).

10. Ollivier, H. \& Zurek, W. H. Quantum Discord: A Measure of the Quantumness of Correlations. Phys. Rev. Lett. 88, 017901 (2001)

11. Piani, M., Horodecki, P. \& Horodecki, R. No-Local-Broadcasting Theorem for Multipartite Quantum Correlations. Phys. Rev. Lett. 100, 090502 (2008).

12. Wu, S., Poulsen, U. V. \& Mølmer, K. Correlations in local measurements on a quantum state, and complementarity as an explanation of nonclassicality. Phys. Rev. A 80, 032319 (2009).

13. Oppenheim, J., Horodecki, M., Horodecki, P. \& Horodecki, R. Thermodynamical Approach to Quantifying Quantum Correlations. Phys. Rev. Lett. 89, 180402 (2002)

14. Horodecki, M., Horodecki, K., Horodecki, P., Horodecki, R., Oppenheim, J., Sen(De), A. \& Sen, U. Local Information as a Resource in Distributed Quantum Systems. Phys. Rev. Lett. 90, 100402 (2003).

15. Luo, S. Using measurement-induced disturbance to characterize correlations as classical or quantum. Phys. Rev. A 77, 022301 (2008).

16. Modi, K., Paterek, T., Son, W., Vedral, V. \& Williamson, M. Unified View of Quantum and Classical Correlations. Phys. Rev. Lett. 104, 080501 (2010).

17. Dakic, B., Vedral, V. \& Brukner, C. Necessary and Sufficient Condition for Nonzero Quantum Discord. Phys. Rev. Lett. 105, 190502 (2010).

18. Luo, S. \& Fu, S. Geometric measure of quantum discord. Phys. Rev. A 82, 034302 (2010).

19. Adesso, G. \& Datta, A. Quantum versus Classical Correlations in Gaussian States. Phys. Rev. Lett. 105, 030501 (2010).

20. Giorda, P. \& Paris, M. G. A. Gaussian Quantum Discord. Phys. Rev. Lett. 105 020503 (2010)

21. Modi, K., Brodutch, A., Cable, H., Paterek, T. \& Vedral, V. The classical-quantum boundary for correlations: Discord and related measures. Rev. Mod. Phys. 84, 1655-1707 (2012).
22. Streltsov, A. \& Zurek, W. H. Quantum Discord Cannot Be Shared. Phys. Rev. Lett. 111, 040401 (2013)

23. Coles, P. J. Unification of different views of decoherence and discord. Phys. Rev. A 85, 042103 (2012)

24. Aaronson, B., Lo Franco, R., Compagno, G. \& Adesso, G. Hierarchy and dynamics of trace distance correlations. New J. Phys. 15, 093022 (2013)

25. Bell, J. S. On the Einstein Podolsky Rosen paradox. Physics 1, 195-200 (1964).

26. Genovese, M. Research on hidden variable theories: A review of recent progresses. Phys. Rep. 413, 319-396 (2005).

27. Groisman, B., Popescu, S. \& Winter, A. Quantum, classical, and total amount of correlations in a quantum state. Phys. Rev. A 72, 032317 (2005).

28. Wu, S. Correlation measures in bipartite states and entanglement irreversibility. Phys. Rev. A 86, 012329 (2012).

29. Henderson, L. \& Vedral, V. Classical, quantum and total correlations. J. Phys. A 34, 6899-6905 (2001).

30. Ivanovic, I. D. Geometrical description of quantum state determination. J. Phys. A 14, 3241-3245 (1981)

31. Ivanovic, I. D. Unbiased projector basis over $C^{3}$. Phys. Lett. A 228, 329-334 (1997).

32. Wootters, W. K. \& Fields, B. D. Optimal state-determination by mutually unbiased measurements. Ann. Phys. 191, 363-381 (1989).

33. Bandyopadhyay, S., Boykin, P. O., Roychowdhury, V. \& Vatan, F. A new proof for the existence of mutually unbiased bases. Algorithmica 34, 512-528 (2002).

34. Durt, T., Englert, B.-G., Bengtsson, I. \& Zyczkowski, K. On mutually unbiased bases. Int. J. Quant. Inf. 8, 535-640 (2010).

35. Werner, R. F. Quantum states with Einstein-Podolsky-Rosen correlations admitting a hidden-variable model. Phys. Rev. A 40, 4277-4281 (1989).

36. Wootters, W. K. Entanglement of formation and concurrence. Quant. Inf. \& Comp. 1, 27-44 (2001).

37. Berta, M., Christandl, M., Colbeck, R., Renes, J. M. \& Renner, R. The uncertainty principle in the presence of quantum memory. Nat. Phys. 6, 659-662 (2010).

38. Streltsov, A., Kampermann, H. \& Bruß, D. Behavior of quantum correlations under local noise. Phys. Rev. Lett. 107, 170502 (2011).

\section{Acknowledgments}

The authors like to thank Caslav Brukner and Yuchun Wu for valuable discussions. S.W. acknowledges support from the NSFC via Grant 11275181, the CAS and the National Fundamental Research Program. Z.M. acknowledges support from the NSFC via Grant 11371247. Z.C. acknowledges support from the NSFC via Grant 11201427. S.Y. acknowledges support from the National Research Foundation and Ministry of Education (Singapore) via Grant WBS: R-710-000-008-271.

\section{Author contributions}

S.W. introduced the idea and definitions, calculated the examples and wrote the manuscript. S.Y. proved the inequalities relations. Z.M. and Z.C. preformed analyses and discussed the results. All authors reviewed the manuscript.

\section{Additional information}

Competing financial interests: The authors declare no competing financial interests. How to cite this article: Wu, S.J., Ma, Z.H., Chen, Z.H. \& Yu, S.X. Reveal quantum correlation in complementary bases. Sci. Rep. 4, 4036; DOI:10.1038/srep04036 (2014)

cc)(1) (2) This work is licensed under a Creative Commons AttributionNonCommercial-ShareAlike 3.0 Unported license. To view a copy of this license, visit http://creativecommons.org/licenses/by-nc-sa/3.0 